Author affiliations appear at the end of this article.

Published at ascopubs.org/journal/jco on November 21, 2016.

Support information appears at the end of this article.

Results were presented in part at the 2014 American Society of Hematology Annual Meeting in San Francisco, CA, December 6-9, 2014.

Corresponding author: Eric J. Chow, MD, $\mathrm{MPH}$, Fred Hutchinson Cancer Research Center, PO Box 19024, M4-C308, Seattle WA 98109; e-mail: ericchow@u. washington.edu.

(C) 2016 by American Society of Clinical Oncology

0732-183X/17/3503w-306w/\$20.00

\title{
Morbidity and Mortality Differences Between Hematopoietic Cell Transplantation Survivors and Other Cancer Survivors
}

Eric J. Chow, Kara L. Cushing-Haugen, Guang-Shing Cheng, Michael Boeckh, Nandita Khera, Stephanie J. Lee, Wendy M. Leisenring, Paul J. Martin, Beth A. Mueller, Stephen M. Schwartz, and K. Scott Baker

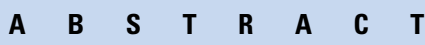

\section{Purpose}

To compare the risks of serious health outcomes among hematopoietic cell transplantation (HCT) survivors versus a matched population of patients with cancer who did not undergo HCT, where the primary difference may be exposure to HCT.

\section{Methods}

Two-year HCT survivors treated at a comprehensive cancer center from 1992 through 2009 who were Washington State residents ( $n=1,792 ; 52 \%$ allogeneic and $90 \%$ hematologic malignancies) were frequency matched by demographic characteristics and underlying cancer diagnosis (as applicable) to non-HCT 2-year cancer survivors, using the state cancer registry $(n=5,455)$ and the general population ( $n=16,340$ ) using driver's license files. Late outcomes for all three cohorts were ascertained from the state hospital discharge and death registries; subsequent cancers were ascertained from the state cancer registry.

\section{Results}

After median follow-up of 7.1 years, HCT survivors experienced significantly greater rates of hospitalization compared with matched non-HCT cancer survivors (280 v 173 episodes per 1,000 person-years, $P<.001$ ) and greater all-cause mortality (hazard ratio [HR], 1.1; 95\% Cl, 1.01 to 1.3). HCT survivors had more hospitalizations or death with infections (10-year cumulative incidence, $31 \% \mathrm{v} 22 \% ; \mathrm{HR}, 1.4 ; 95 \% \mathrm{Cl}, 1.3$ to 1.6 ) and respiratory complications (cumulative incidence, $27 \% \mathrm{v}$ $20 \% ; \mathrm{HR}, 1.4 ; 95 \% \mathrm{Cl}, 1.2$ to 1.5$)$. Risks of digestive, skin, and musculoskeletal complications also were greater among HCT versus non-HCT cancer survivors. The two groups had similar risks of circulatory complications and second cancers. Both HCT and non-HCT cancer survivors had significantly greater 10-year cumulative incidences of all major organ-system outcomes versus the general population.

\section{Conclusion}

History of HCT was associated with late morbidity and mortality among cancer survivors. In particular, clinicians who care for HCT survivors should be aware of their high rates of late respiratory and infectious complications.

\section{J Clin Oncol 35:306-313. (C) 2016 by American Society of Clinical Oncology}

\section{INTRODUCTION}

The use of autologous and allogeneic hematopoietic cell transplantation (HCT) to treat highrisk malignancies has increased markedly during the past 25 years. ${ }^{1}$ Among $\geq 2$-year HCT survivors, more than $70 \%$ remain long-term survivors, and nearly 250,000 HCT survivors (estimated 60\% autologous; $40 \%$ allogeneic) are predicted to be living in the United States by the year $2020 .^{2}$ Thus, it becomes important to clarify the patterns of late morbidity and mortality in this population., Most studies of late serious health outcomes following HCT have focused on original disease relapse, chronic graft versus host disease (GVHD), and second cancers. The incidence of other serious outcomes among HCT survivors remains less well described, although rates of serious cardiopulmonary disease, long-term infectious complications, and overall rates of serious chronic conditions also are greater than in the general population. ${ }^{5-10}$ Many previous studies were limited by small sample size, potential response or survival biases, or loss to follow-up when they depended on patient self-report and data from transplant centers. 
Furthermore, it is unclear whether HCT survivors are at greater risk of adverse long-term health conditions compared with other cancer survivors.

To address these limitations, we linked a large HCT cohort with state hospital and death registry data and compared the burden of hospitalizations and deaths among $\geq 2$-year HCT survivors with those experienced by both the general population and a non-HCT cancer survivor cohort derived from the state cancer registry and matched on the same underlying diagnoses. These data provided population-based estimates of the absolute and relative risks of serious health outcomes affecting each group and allowed us to examine whether HCT survivors have risks beyond those experienced by cancer survivors who did not receive HCT.

\section{METHODS}

\section{HCT Survivor and Comparison Cohorts}

HCT survivors were Washington State residents treated at the Fred Hutchinson Cancer Research Center (FHCRC), a National Cancer Institute-designated comprehensive cancer center and the only accredited institution that performs allogeneic HCT in Washington (a few other institutions perform autologous HCT only). The Fred Hutchinson Cancer Research Center (FHCRC) database was screened to identify patients who received any HCT from 1992 through $2009(\mathrm{n}=7,108)$, were alive $\geq 2$ years post-transplant $(n=4,081)$, were state residents at the time of transplantation $(\mathrm{n}=1,929)$, and received transplantation for a malignant condition $(n=1,792)$. Race and ethnicity, clinically recorded pretransplant diagnosis, and transplant-related exposures (eg, donor type, stem-cell source, conditioning regimen, chronic GVHD requiring systemic immunosuppressive treatment) were obtained from the FHCRC database.

A comparison cohort of patients with cancer diagnoses was identified from the Washington State Cancer Registry (non-HCT group). After excluding subjects who were FHCRC HCT survivors, the remaining state residents who had an initial cancer diagnosis from 1992 through 2009 and survived $\geq 2$ years were randomly selected and frequency matched to HCT survivors at a 3:1 ratio by sex, age at cancer diagnosis or HCT (10-year age increments plus age $<20$ years and $\geq 60$ years), year of cancer diagnosis or HCT (2-year increments), and cancer diagnosis group $(\mathrm{n}=5,455$; Data Supplement).

A separate, population-based noncancer comparison cohort was available from the Washington State Department of Licensing (DOL) files for 1992 through 2009 that did not overlap with either the HCT or nonHCT groups. Subjects had been randomly selected from these files to be frequency matched $(10: 1$ ratio; $n=16,340)$ based on their age at and year of driver's license issuance or renewal to $\geq 2$-year FHCRC HCT survivors who were state residents and $\geq 16$ years of age at HCT, by sex, age at and year of HCT ( $n=1,634$; including 68 HCT survivors who had received transplantation for nonmalignant diseases). State death records were screened to ensure that the comparison subjects did not die within 2 years of licensing.

\section{Outcomes Ascertainment}

For hospitalization data, we used the Washington State Comprehensive Hospital Abstract Reporting System, a database of all hospital discharges from non-federal facilities. This database contains up to nine diagnosis codes (International Classification of Diseases, 9th revision [ICD]) for each hospitalization during the years of study. Identifiers allowed longitudinal tracking of individuals across multiple hospitalizations and linkage to outside data sources. For mortality, we used the state death registry, which recorded the primary and up to six contributing causes of death (ICD-10) and includes deaths of residents who died in neighboring states. Similar to our previous work, we used a sequential deterministic linkage strategy to link survivors and comparison subjects to hospital discharge and death records from 1992 through $2011 .^{11,12}$ Variables available for linkage included components of names, birth date, sex, zip code, county of residence, date of HCT and corresponding hospitalization, and date of death (if applicable).

We assigned linked ICD-9 and equivalent ICD-10 codes to major outcome categories, primarily based on organ systems (Data Supplement), occurring $\geq 2$ years after the index date (ie, transplant for HCT group, cancer diagnosis for non-HCT group, and drivers' licensing date for DOL group). For each category, we also examined infection- and noninfectionassociated codes separately. To ascertain subsequent cancers, the state cancer registry was used to identify new cancers occurring among HCT and non-HCT cancer survivors.

\section{Statistical Analysis}

For each outcome, we estimated the 10-year cumulative incidence and associated differences among HCT and comparison cohorts starting 2 years from the index date. Follow-up of individuals without events was censored on December 31, 2011 or earlier if they died of any cause other than the specific outcome category examined. ${ }^{13}$ Analysis of pregnancy outcomes was restricted to women 15 to 44 years of age during the study period. Mental health outcomes were available only for individuals $\geq 18$ years of age because of state policy.

We used Cox proportional hazards models with age as the time scale, left-truncated with individuals entering the analysis 2 years from their index date, to estimate the hazard ratios (HRs) and 95\% CIs for each outcome adjusted for sex, year of index event, and underlying diagnosis group for HCT versus non-HCT cancer survivors. ${ }^{14}$ In analyses restricted to HCT recipients, using a time since transplant scale, we also examined the contributions of race and ethnicity, age at transplantation, donor type, chronic GVHD, and relapse of the original disease within the first 2 years after HCT. Subanalyses examined these relationships among $\geq 5$-year survivors. History of total body irradiation (TBI; none, $<10 \mathrm{~Gy}$, and $\geq 10$ Gy) was examined but was not included in final models because we did not have information regarding radiotherapy received before referral for HCT. Global tests of proportionality based on Schoenfeld residuals were used to ensure that proportional hazards assumptions were met $(P \geq .05)$; covariates with nonproportional hazards were treated as time-varying. ${ }^{14}$ Finally, given that the burden of some outcomes may be better described by a model that allows multiple events rather than time-to-first event, we also applied Poisson regression to estimate the relative risks (RRs) for multiple infections and respiratory complications between cohorts during the observation period. ${ }^{15}$ All analyses were performed using STATA, version 14 (StataCorp, College Station, TX, http://www.stata.com/).

\section{RESULTS}

The sex and age distribution among all three groups was similar by design (Table 1). The median age at HCT was 47 years (interquartile range, 31 to 57 ), similar to the median ages of the non-HCT group at cancer diagnosis ( 49 years; interquartile range, 34 to 58 years) and the DOL group ( 48 years; interquartile range, 37 to 57 years). Among individuals not known to be deceased, the three groups had similar median follow-up duration (HCT: 7.1 years; non-HCT: 7.2 years; and DOL: 7.7 years; range, 2 to 20 years for all three groups). Within the HCT group, the median time from original disease diagnosis to transplantation was 9.3 months (interquartile range, 5.7 to 19.7 months). A total of $143 \mathrm{HCT}$ survivors $(8.0 \%)$ also received a subsequent transplant before reaching the 2-year survival landmark. 


\begin{tabular}{|c|c|c|c|c|c|c|}
\hline Characteristic & No. & $\%$ & No. & $\%$ & No. & $\%$ \\
\hline \multicolumn{7}{|l|}{ Race/ethnicity } \\
\hline White non-Hispanic & 1,504 & 83.9 & 4,543 & 83.3 & - & \\
\hline Black & 40 & 2.2 & 202 & 3.7 & - & \\
\hline Other/unknown & 92 & 5.1 & 199 & 3.6 & - & \\
\hline \multicolumn{7}{|l|}{ Age at index date, years $\ddagger$} \\
\hline$<20$ & 277 & 15.5 & 767 & 14.1 & 570 & 3.5 \\
\hline $20-39$ & 376 & 21.0 & 934 & 17.1 & 4,100 & 25.1 \\
\hline $40-59$ & 843 & 47.0 & 2,590 & 47.5 & 8,660 & 53.0 \\
\hline$\geq 60$ & 296 & 16.5 & 1,164 & 21.3 & 3,010 & 18.4 \\
\hline \multicolumn{7}{|l|}{ Year of index event } \\
\hline \multicolumn{7}{|l|}{ Donor type } \\
\hline Autologous/isogeneic & 867 & 48.4 & - & & - & \\
\hline Related allogeneic & 503 & 28.1 & - & & - & \\
\hline Unrelated allogeneic & 422 & 23.5 & - & & - & \\
\hline \multicolumn{7}{|l|}{ History of total body irradiation } \\
\hline None & 969 & 54.1 & - & & - & \\
\hline$<10 \mathrm{~Gy}$ & 221 & 12.3 & - & & - & \\
\hline$\geq 10 \mathrm{~Gy}$ & 602 & 33.6 & - & & - & \\
\hline History of chronic GVHD\| & 642 & 36.1 & - & & - & \\
\hline History of cancer relapse after index date & 434 & 24.2 & - & & - & \\
\hline Died during the follow-up period & 388 & 21.7 & 997 & 18.3 & 581 & 3.6 \\
\hline
\end{tabular}

The 10-year cumulative incidence of any hospitalization or death related to all major organ-system outcomes was significantly different $(P<.05)$ between the HCT and DOL groups (Fig 1; Data Supplement). Differences in cumulative incidences between the HCT and non-HCT groups were less pronounced. The HCT group notably had a $30.6 \%$ cumulative incidence of infectious complications (difference $v$ non-HCT: $8.7 \%$, 95\% CI 5.9\% to $11.6 \%$ ) and a $26.8 \%$ incidence of any respiratory complications (difference $v$ non-HCT: $6.9 \%, 95 \%$ CI $4.2 \%$ to $9.6 \%$ ). The 10 -year cumulative incidences of nervous system, circulatory, and genitourinary

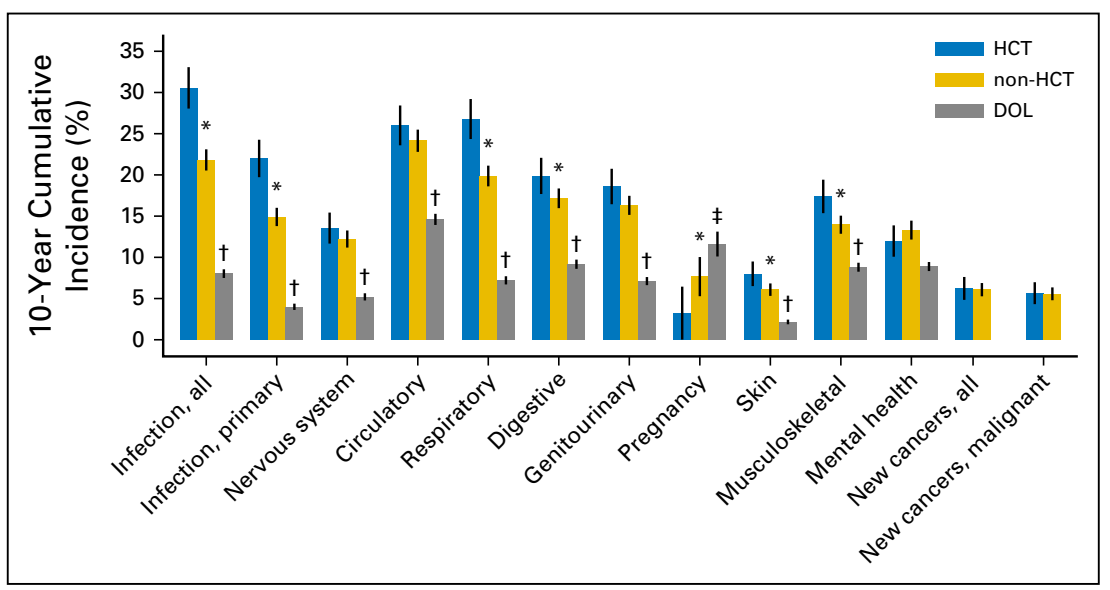

Fig 1. Ten-year cumulative incidence with $95 \% \mathrm{Cls}$ (spikes) of major outcomes among $\geq 2$-year HCT survivors and comparison cohorts (non-HCT cancer survivors; general population, DOL). Incidence of pregnancy was limited to women ages 15 to 44 years during the observation period. Subsequent new cancers were only ascertained among HCT and non-HCT cancer survivors. ${ }^{*} P<.05$ for differences between HCT $v$ non-HCT. $\uparrow P<.05$ for differences between HCT $v \mathrm{DOL}$ and non-HCT $v \mathrm{DOL}$, limited to individuals age $\geq 16$ years. $\ddagger P<.05$ for difference between HCT $\vee$ DOL only. DOL, Department of Licensing; HCT, hematopoietic cell transplantation. 
complications, mental health outcomes, and subsequent new cancers were similar between the HCT and non-HCT groups. Among women ages 15 to 44 years, the incidence of pregnancyrelated hospitalization was lower in the HCT group versus the nonHCT group (difference, $-4.4 \%, 95 \% \mathrm{CI},-8.4$ to $-0.4 \%$ ).

Adjusted analyses starting at the 2-year landmark showed that certain risks were notably higher in the HCT group versus the nonHCT group (Table 2). These included primary infections (HR, 1.4; 95\% CI, 1.2 to 1.6 ), respiratory complications (HR, 1.3; 95\% CI, 1.2 to 1.5$)$, and death from any cause (HR, $1.1 ; 95 \% \mathrm{CI}, 1.01$ to 1.3). Risks of hospitalization for nervous system, circulatory, digestive, and genitourinary complications, and mental health conditions were similar (HR range, 0.9 to $1.1 ; P>.05$ ). The risk of subsequent cancers, based on state cancer registry data, also was similar in the two groups. For most outcomes, the relative hazards associated with HCT tended to increase for most outcomes beyond 5 years of follow-up (Table 2). Among $\geq 2$-year female survivors, the likelihood of pregnancy-associated hospitalizations was lower in the HCT group $(n=5)$ versus the non-HCT group $(n=46$; HR, $0.1,95 \%$ CI, 0.01 to 0.6 for ages 15 to 29 years; HR, $0.5,95 \%$ CI, 0.1 to 1.5 for ages 30 to 44 years). Finally, comparisons of HCT with non-HCT survivors adjusted for donor type (ie, autologous, allogeneic) generally showed similar results (Data Supplement). Results restricted to hematologic malignancies also generally showed similar results (data not shown), although risks of specific outcomes did appear to vary somewhat when cancer diagnostic groups were examined individually (Data Supplement).

Among organ system-specific infection categories, the HCT group was more likely to experience infections of the nervous system (HR, 2.5; 95\% CI, 1.4 to 4.6 ; most commonly meningitis), respiratory system (HR, 1.6; 95\% CI, 1.4 to 1.9 ; most commonly pneumonia), and the digestive tract (HR, 1.7; 95\% CI, 1.2 to 2.53; most commonly oral, gastrointestinal, and biliary infections) compared with the non-HCT group. The risk of noninfectious respiratory complications (ie, chronic obstructive pulmonary disease, asthma, pulmonary fibrosis, and pneumonopathy) also was greater in the HCT group versus the non-HCT group (HR, 1.3; $95 \% \mathrm{CI}, 1.1$ to 1.5$)$. Poisson models that allowed for multiple events confirmed greater risks of primary infections (RR, 1.6; 95\% CI, 1.3 to 1.9 ) and respiratory complications (RR, 1.7; 95\% CI, 1.4 to 2.0) in the HCT group compared with the non-HCT group.

The HCT group overall had a significantly greater hospitalization rate compared with the non-HCT group (280 episodes per 1,000 person-years $v 173$ episodes per 1,000 person years; $P<.001$ ). When selected hospitalization discharge diagnoses were examined, the proportions of patients with a primary infectious, respiratory, or musculoskeletal condition diagnosis appeared similar for the HCT and non-HCT groups (Table 3; Data Supplement).

Among subsequent cancers reported within the HCT group ( $\mathrm{n}=128$ cancers) and non-HCT group ( $\mathrm{n}=438$ cancers), 107 $(83.6 \%)$ and $304(69.4 \%)$ cancers occurred after 1 year, respectively, with $>85 \%$ in both groups being malignant versus benign or in situ tumors (Data Supplement). Among cancers diagnosed in at least four individuals after the 2-year landmark, the HCT group had greater risks of oral cancers (HR, 5.4; 95\% CI, 1.6 to 18.4 ) and skin cancers compared with the non-HCT group (HR, 4.9; $95 \%$ CI, 1.9 to 12.7 ; primarily melanoma, because squamous and basal cell carcinomas were not ascertained).

Within the HCT group, the risks of morbidity and mortality outcomes for most outcome categories were significantly and independently associated with chronic GVHD and relapse of the original disease, but not with donor type (Table 4). In analyses restricted to events occurring $\geq 5$ years after the index transplantation, a history of chronic GVHD remained significantly associated with greater risks of primary infections and respiratory and genitourinary conditions. Chronic GVHD also appeared to be associated with greater risks of new cancers, but HR estimates were imprecise and were not statistically significant. When history of TBI was added to the analyses (data not shown), those who had received TBI $<10$ Gy experienced a greater burden of infectious

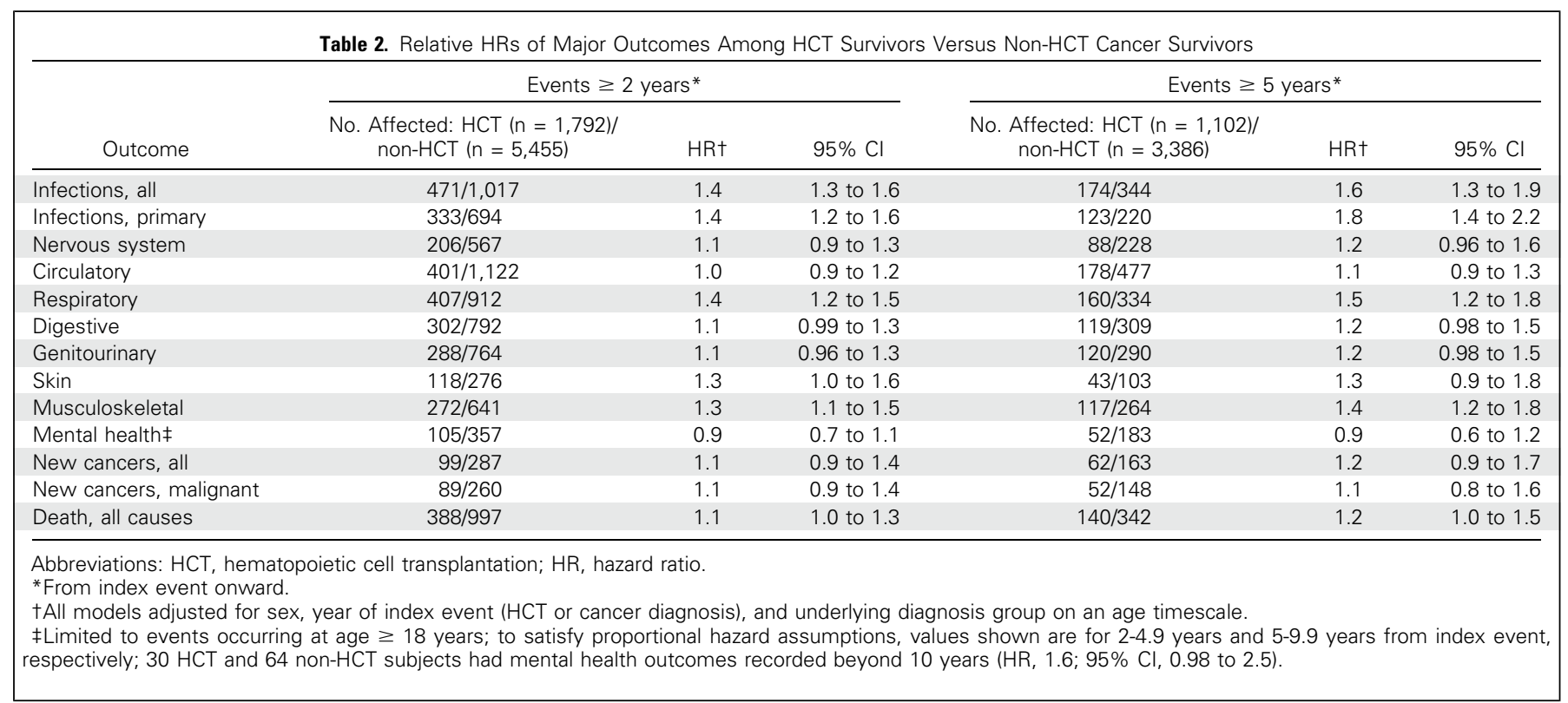




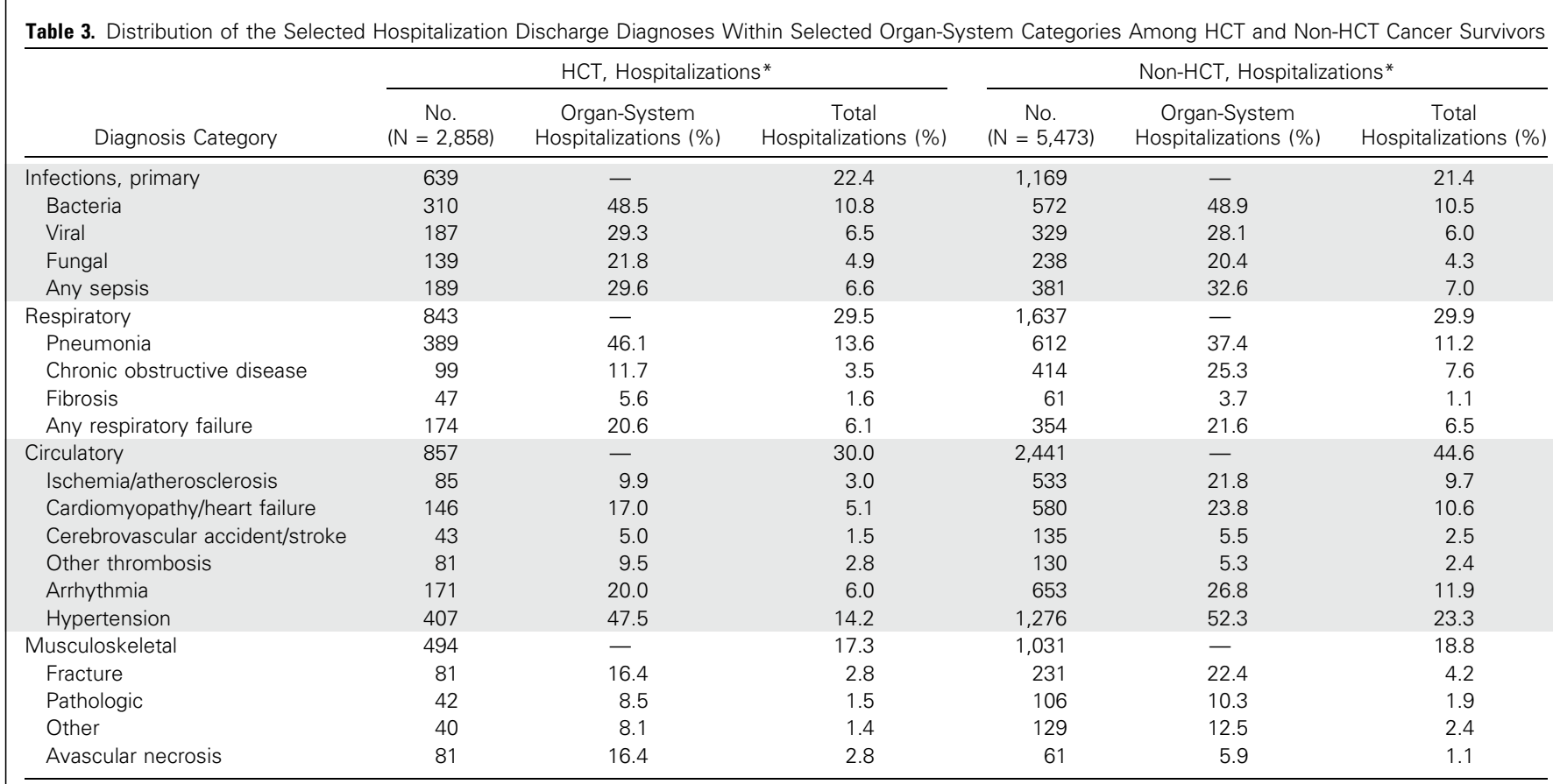

Abbreviations: - , not applicable; HCT, hematopoietic cell transplantation.

*Limited to hospitalizations occurring $\geq 2$ years after index date. Each hospitalization may contain multiple codes, and some participants had multiple hospitalizations. Specific codes corresponding to each category are listed in the Data Supplement.

and respiratory complications 2 years after transplant versus survivors without TBI (HR range, 1.6 to $1.7 ; P<.01$ ). In contrast, survivors treated with $\mathrm{TBI} \geq 10 \mathrm{~Gy}$ did not experience more infectious or respiratory complications than those without TBI, but had a greater risk of new cancers after 2 years (HR range, 2.3 to $2.5 ; P<.01$ ). Receipt of more than one HCT before 2-year survival was not associated with an increased hazard of any subsequent outcome (data not shown).

\section{DISCUSSION}

We found that HCT survivors experienced a significantly greater burden of hospitalizations and mortality compared with matched non-HCT cancer survivors. Disparities in infectious and respiratory complications were marked, whereas the burden of circulatory disease, mental health diagnoses, and second cancers appeared similar between

\begin{tabular}{|c|c|c|c|c|c|c|c|c|c|c|c|c|}
\hline \multirow[b]{3}{*}{ Outcome } & \multicolumn{6}{|c|}{ Events $\geq 2$ Years } & \multicolumn{6}{|c|}{ Events $\geq 5$ Years } \\
\hline & \multicolumn{2}{|c|}{$\begin{array}{l}\text { Allogeneic } v \\
\text { Autologous }\end{array}$} & \multicolumn{2}{|c|}{$\begin{array}{c}\text { Chronic GVHD v } \\
\text { Nonet }\end{array}$} & \multicolumn{2}{|c|}{$\begin{array}{l}\text { Original Disease } \\
\text { Relapse } v \text { None }\end{array}$} & \multicolumn{2}{|c|}{$\begin{array}{l}\text { Allogeneic } v \\
\text { Autologous }\end{array}$} & \multicolumn{2}{|c|}{$\begin{array}{c}\text { Chronic GVHD v } \\
\text { Nonet }\end{array}$} & \multicolumn{2}{|c|}{$\begin{array}{l}\text { Original Disease } \\
\text { Relapse } v \text { None }\end{array}$} \\
\hline & $\mathrm{HR}$ & $95 \% \mathrm{Cl}$ & $\mathrm{HR}$ & $95 \% \mathrm{Cl}$ & $\mathrm{HR}$ & $95 \% \mathrm{Cl}$ & $\mathrm{HR}$ & $95 \% \mathrm{Cl}$ & $\mathrm{HR}$ & $95 \% \mathrm{Cl}$ & $\mathrm{HR}$ & $95 \% \mathrm{Cl}$ \\
\hline Infection, all & 0.9 & 0.7 to 1.3 & 1.5 & 1.1 to 2.0 & 2.4 & 1.9 to 3.0 & 0.8 & 0.5 to 1.3 & 1.2 & 0.8 to 2.0 & 2.3 & 1.6 to 3.2 \\
\hline Infection, primary & 0.8 & 0.5 to 1.2 & 1.7 & 1.2 to 2.4 & 2.8 & 2.1 to 3.6 & 0.5 & 0.3 to 0.96 & 2.0 & 1.04 to 3.9 & 2.6 & 1.8 to 3.8 \\
\hline Nervous system & 0.5 & 0.3 to 0.8 & 2.5 & 1.4 to 4.3 & 2.6 & 1.8 to 3.6 & 0.4 & 0.2 to 0.9 & 2.0 & 0.8 to 4.7 & 2.4 & 1.5 to 3.8 \\
\hline Circulatory & 0.7 & 0.5 to 1.0 & 1.6 & 1.1 to 2.2 & 2.0 & 1.5 to 2.5 & 0.9 & 0.6 to 1.5 & 1.3 & 0.8 to 2.1 & 2.1 & 1.5 to 3.0 \\
\hline Respiratory & 0.8 & 0.6 to 1.1 & 1.7 & 1.2 to 2.4 & 2.0 & 1.6 to 2.6 & 0.6 & 0.3 to 1.1 & 2.0 & 1.1 to 3.5 & 2.2 & 1.5 to 3.1 \\
\hline Digestive & 0.7 & 0.5 to 1.1 & 1.6 & 1.1 to 2.4 & 2.3 & 1.7 to 3.0 & 0.7 & 0.4 to 1.3 & 1.6 & 0.9 to 3.0 & 2.1 & 1.4 to 3.2 \\
\hline Genitourinary & 0.8 & 0.5 to 1.2 & 1.7 & 1.2 to 2.6 & 2.2 & 1.7 to 3.0 & 0.6 & 0.3 to 1.3 & 2.2 & 1.1 to 4.2 & 2.3 & 1.5 to 3.5 \\
\hline Skin & 1.1 & 0.6 to 1.9 & 1.5 & 0.9 to 2.7 & 2.3 & 1.4 to 3.6 & 1.3 & 0.5 to 3.0 & 0.9 & 0.4 to 2.1 & 2.3 & 1.2 to 4.5 \\
\hline Musculoskeletal & 0.8 & 0.5 to 1.3 & 1.9 & 1.2 to 2.9 & 1.4 & 0.99 to 2.0 & 0.7 & 0.4 to 1.3 & 1.4 & 0.8 to 2.5 & 2.1 & 1.4 to 3.2 \\
\hline Mental health $\ddagger$ & 1.0 & 0.6 to 1.5 & 0.9 & 0.9 to $1.0 \S$ & 2.4 & 1.6 to 3.4 & 1.3 & 0.7 to 2.4 & 0.6 & 0.3 to 1.2 & 1.6 & 0.9 to 2.7 \\
\hline New cancers, all & 0.7 & 0.4 to 1.5 & 1.7 & 0.8 to 3.5 & 1.1 & 0.6 to 2.1 & 0.6 & 0.2 to 1.6 & 2.5 & 0.9 to 6.4 & 0.9 & 0.4 to 1.8 \\
\hline New cancers, malignant & 0.8 & 0.4 to 1.7 & 1.4 & 0.6 to 2.8 & 1.3 & 0.7 to 2.4 & 0.6 & 0.2 to 1.7 & 2.3 & 0.8 to 6.7 & 1.1 & 0.5 to 2.3 \\
\hline
\end{tabular}

Abbreviations: GVHD, graft versus host disease; HR, hazard ratio

* Models all adjusted for sex, race and ethnicity, and age at and year of transplantation, donor type, history of chronic GVHD, and history of original disease relapse. tLimited to those who required systemic immunosuppressive therapy.

fLimited to those age $\geq 18$ years.

§Chronic GVHD modeled as time-varying to meet proportional hazard assumptions. 
these two groups. In general, differences were magnified when the analysis was limited to adverse events occurring $\geq 5$ years after the index date, thereby reducing the contribution related to the acute toxicities of treatment, original disease relapse, and active chronic GVHD. A major strength of this study is its reliance on prospectively coded and population-based data sources free of response biases.

We previously used statewide registry data to examine cardiovascular hospitalizations and mortality and found greater absolute and relative risks when HCT survivors were compared with the general population. ${ }^{11}$ The current analysis is more nuanced and showed that the risks for cardiovascular disease, at least overall, may not differ between HCT survivors and matched non-HCT cancer survivors. Similarly, we did not find a significantly different overall risk of second cancers, although specific cancers affecting the skin (primarily melanoma) and the oral cavity were more common among HCT survivors than non-HCT cancer survivors. Exposures unique to HCT survivors, such as chronic GVHD, may be responsible for the greater risks of these specific tumor types. ${ }^{16}$ Such distinctions are important because clinicians have hypothesized that HCT survivors may be at greater risk for long-term health complications compared with other cancer survivors, and may require additional surveillance or health counseling beyond those recommended for other cancer survivors. Our analysis provided additional context regarding those organ systems and outcome categories where this hypothesis may be correct and others where this hypothesis may be less compelling.

Few comprehensive analyses have compared HCT with non-HCT cancer survivors. A report examining survivors of childhood HCT compared with non-HCT survivors of childhood hematologic malignancies showed that HCT survivors had a nearly four-times greater risk of self-reported severe or life-threatening chronic conditions, including second cancers, endocrine conditions, and musculoskeletal conditions. $^{17}$ TBI and any history of chronic GVHD (active or resolved) were associated with the development of more severe chronic conditions. Relative differences in second-cancer risk between our studies could be related, in part, to the composition of study participants (ie, childhood $v$ mostly adult-onset cancer survivors). It also is possible that the experiences of survivors treated at other institutions and other geographic regions may differ from our findings.

Studies also have shown that HCT survivors often have pulmonary dysfunction, including both restrictive and obstructive disease. ${ }^{18,19}$ A subset of allogeneic survivors develop bronchiolitis obliterans syndrome, which is characterized by chronic irreversible airflow obstruction and is associated with chronic GVHD. ${ }^{20}$ Other risk factors for pulmonary dysfunction after HCT include a history of TBI and respiratory viral infections. ${ }^{18,19}$ Although most studies suggest that pulmonary function abnormalities increase over time, ${ }^{19,21}$ some degree of recovery may be possible. ${ }^{22}$ Current guidelines recommend that survivors of autologous HCT and allogeneic HCT be screened annually with a history and physical examination for pulmonary dysfunction, ${ }^{3}$ with consideration of more frequent pulmonary function testing among survivors with chronic GVHD. ${ }^{23}$ Our results would support this recommendation.

Immunity is altered as a direct consequence of HCT, and immune reconstitution may be further disrupted by GVHD and long-term immunosuppression. ${ }^{24-26}$ Although current guidelines on antimicrobial prophylaxis and reimmunization primarily focus on patients with chronic GVHD, ${ }^{3}$ our results suggest that even those without chronic GVHD, including autologous HCT survivors, remain at increased risk long term. This finding corroborates prior studies showing that infection remains a leading cause of death, even in the absence of GVHD, among $\geq 2$-year allogeneic HCT survivors, including $\geq 5$ or $\geq 10$-year survivors. ${ }^{8,10}$ Studies of pediatric autologous HCT recipients have shown that immune reconstitution, in particular T-cell recovery, may be abnormal even 2 or more years after HCT. $^{27}$

Other findings in our study include a reduced likelihood of pregnancy-related hospitalizations compared with non-HCT female cancer survivors, but an overall similar risk of mental health conditions between the two groups. Both TBI and high-dose alkylating agents (eg, cyclophosphamide, busulfan) used as part of the conditioning regimen are associated with adverse effects on fertility. ${ }^{28-30}$ Whereas HCT survivors had similar rates of mental health conditions compared with non-HCT survivors, the burden of these conditions was greater compared with the general population. A similar increased burden among HCT survivors versus siblings has been reported. ${ }^{31}$ However, psychological health may improve over time for many HCT survivors. ${ }^{32}$

Our results should be interpreted with some considerations. Although we specifically matched HCT with non-HCT cancer survivors by original diagnosis, sex, and age at and year of HCT or cancer diagnosis, we could not account for pre-existing comorbidities or cancer treatment exposures that occurred before HCT and those received by the non-HCT group. Some of the differences between groups may be due to more advanced disease and more pre-HCT cancer treatment exposures in HCT recipients. At the same time, prerequisites for adequate organ function may be more stringent for HCT candidates, ${ }^{33}$ which may minimize differences between HCT and non-HCT survivors. Future analyses that focus on specific outcome categories could attempt to reconstruct exposures such as radiation and chemotherapy received before HCT, as well as pre-existing comorbidities.

Although a strength of our analysis is the lack of response bias, misclassification of outcomes can still occur when using registry and administrative databases. However, any misclassification should apply similarly across groups and would be expected to bias results toward the null. In general-population studies, the specificity of hospital discharge and other administrative datasets is typically more than $90 \%$, but sensitivity can be more variable compared with chart review. ${ }^{34-38}$ However, longitudinal hospitalization data of the type we used can improve sensitivity without reducing specificity. ${ }^{35}$ Finally, because HCT survivors were hospitalized more frequently than non-HCT cancer survivors, they would have had more opportunities to have diagnoses recorded. We did not find that HCT survivors had a consistently greater burden of all late outcomes compared with non-HCT survivors, arguing against systematic measurement bias. Even so, less severe conditions that did not result in hospitalizations would not have been ascertained in this analysis, but may still affect quality of life.

In summary, we found that HCT survivors were generally at greater risk for all categories of late outcomes compared with the general population. Differences between HCT survivors and matched non-HCT cancer survivors were less pronounced, with the exception of late infections and respiratory (both infectious and noninfectious) complications. Clinicians who care for long-term survivors of HCT should be aware of comprehensive surveillance guidelines available for this high-risk population. ${ }^{3,4,16}$ Future work to identify more specific risk factors associated with late infections and respiratory complications may help to further refine these guidelines and identify new prevention strategies. 
AUTHORS' DISCLOSURES OF POTENTIAL CONFLICTS OF INTEREST

Disclosures provided by the authors are available with this article at ascopubs.org/journal/jco.

\section{AUTHOR CONTRIBUTIONS}

Financial support: Eric J. Chow, Paul J. Martin

Administrative support: Eric J. Chow

Provision of study materials or patients: Eric J. Chow,

Stephen M. Schwartz

Collection and assembly of data: Eric J. Chow

Data analysis and interpretation: All authors

Manuscript writing: All authors

Final approval of manuscript: All authors

Accountable for all aspects of the work: All authors

Conception and design: Eric J. Chow, Wendy M. Leisenring,

Paul J. Martin, Beth A. Mueller, Stephen M. Schwartz, K. Scott Baker

\section{REFERENCES}

1. Appelbaum FR, Forman $S J$, Negrin RS, et al: Uses and growth of hematopoietic cell transplantation, in Appelbaum FR, Forman SJ, Negrin RS et al (eds): Thomas' Hematopoietic Cell Transplantation. 4th Ed. West Sussex, UK, Wiley-Blackwell, 2008, pp 15-21

2. Majhail NS, Tao L, Bredeson C, et al: Prevalence of hematopoietic cell transplant survivors in the United States. Biol Blood Marrow Transplant 19: 1498-1501, 2013

3. Majhail NS, Rizzo JD, Lee SJ, et al: Recommended screening and preventive practices for longterm survivors after hematopoietic cell transplantation. Biol Blood Marrow Transplant 18:348-371, 2012

4. Chow EJ, Anderson L, Baker KS, et al: Late effects surveillance recommendations among survivors of childhood hematopoietic cell transplantation: A Children's Oncology Group report. Biol Blood Marrow Transplant 22:782-795, 2016

5. Socié G, Stone JV, Wingard JR, et al: Long-term survival and late deaths after allogeneic bone marrow transplantation. N Engl J Med 341:14-21, 1999

6. Bhatia $S$, Robison $L L$, Francisco $L$, et al: Late mortality in survivors of autologous hematopoietic-cell transplantation: Report from the Bone Marrow Transplant Survivor Study. Blood 105:4215-4222, 2005

7. Bhatia S, Francisco L, Carter A, et al: Late mortality after allogeneic hematopoietic cell transplantation and functional status of long-term survivors: Report from the Bone Marrow Transplant Survivor Study. Blood 110:3784-3792, 2007

8. Martin PJ, Counts GW, Jr., Appelbaum FR et al: Life expectancy in patients surviving more than 5 years after hematopoietic cell transplantation. J Clin Oncol 28:1011-1016, 2010

9. Sun $C L$, Francisco $L$, Kawashima $T$, et al: Prevalence and predictors of chronic health conditions after hematopoietic cell transplantation: A report from the Bone Marrow Transplant Survivor Study. Blood 116: 3129-3139, quiz 3377, 2010

10. Wingard JR, Majhail NS, Brazauskas R, et al: Long-term survival and late deaths after allogeneic hematopoietic cell transplantation. J Clin Oncol 29: 2230-2239, 2011

11. Chow EJ, Mueller BA, Baker KS, et al: Cardiovascular hospitalizations and mortality among recipients of hematopoietic stem cell transplantation. Ann Intern Med 155:21-32, 2011

12. Herman AA, McCarthy BJ, Bakewell JM, et al: Data linkage methods used in maternally-linked birth and infant death surveillance data sets from the United States (Georgia, Missouri, Utah and Washington State), Israel, Norway, Scotland and Western Australia. Paediatr Perinat Epidemiol 11(suppl 1):5-22, 1997
13. Gooley TA, Leisenring W, Crowley J, et al: Estimation of failure probabilities in the presence of competing risks: New representations of old estimators. Stat Med 18:695-706, 1999

14. Therneau TM, Grambsch PM. Modeling Survival Data: Extending the Cox Model. New York, NY, Springer-Verlag, 2000.

15. Segal MR, Neuhaus JM: Robust inference for multivariate survival data. Stat Med 12:1019-1031, 1993

16. Inamoto $Y$, Shah NN, Savani BN, et al: Secondary solid cancer screening following hematopoietic cell transplantation. Bone Marrow Transplant 50: 1013-1023, 2015

17. Armenian $S H$, Sun $C L$, Kawashima T, et al: Longterm health-related outcomes in survivors of childhood cancer treated with HSCT versus conventional therapy: A report from the Bone Marrow Transplant Survivor Study (BMTSS) and Childhood Cancer Survivor Study (CCSS). Blood 118:1413-1420, 2011

18. Chien JW, Martin PJ, Gooley TA, et al: Airflow obstruction after myeloablative allogeneic hematopoietic stem cell transplantation. Am J Respir Crit Care Med 168:208-214, 2003

19. Hoffmeister PA, Madtes DK, Storer BE, et al: Pulmonary function in long-term survivors of pediatric hematopoietic cell transplantation. Pediatr Blood Cancer 47:594-606, 2006

20. Barker AF, Bergeron $A$, Rom WN, et al: Obliterative bronchiolitis. N Engl J Med 370:1820-1828, 2014

21. Inaba H, Yang J, Pan J, et al: Pulmonary dysfunction in survivors of childhood hematologic malignancies after allogeneic hematopoietic stem cell transplantation. Cancer 116:2020-2030, 2010

22. Jain NA, Pophali PA, Klotz JK, et al: Repair of impaired pulmonary function is possible in very-longterm allogeneic stem cell transplantation survivors. Biol Blood Marrow Transplant 20:209-213, 2014

23. Carpenter PA, Kitko CL, Elad S, et al: National Institutes of Health Consensus Development Project on Criteria for Clinical Trials in Chronic Graft-versusHost Disease: V. The 2014 Ancillary Therapy and Supportive Care Working Group Report. Biol Blood Marrow Transplant 21:1167-1187, 2015

24. Wingard JR, Hsu J, Hiemenz JW: Hematopoietic stem cell transplantation: An overview of infection risks and epidemiology. Infect Dis Clin North Am 24:257-272, 2010

25. Corre $E$, Carmagnat $M$, Busson $M$, et al: Long-term immune deficiency after allogeneic stem cell transplantation: B-cell deficiency is associated with late infections. Haematologica 95: 1025-1029, 2010

26. Bunin $N$, Small $T$, Szabolcs $P$, et al: $N C l$, NHLBI/PBMTC First International Conference on Late Effects After Pediatric Hematopoietic Cell Transplantation: Persistent immune deficiency in pediatric transplant survivors. Biol Blood Marrow Transplant 18:6-15, 2012

27. Kalwak K, Gorczyńska E, Toporski J, et al: Immune reconstitution after haematopoietic cell transplantation in children: Immunophenotype analysis with regard to factors affecting the speed of recovery. $\mathrm{Br} \mathrm{J}$ Haematol 118: 74-89, 2002

28. Chow EJ, Liu W, Srivastava K, et al: Differential effects of radiotherapy on growth and endocrine function among acute leukemia survivors: A childhood cancer survivor study report. Pediatr Blood Cancer 60:110-115, 2013

29. Sanders JE, Hawley J, Levy W, et al: Pregnancies following high-dose cyclophosphamide with or without high-dose busulfan or total-body irradiation and bone marrow transplantation. Blood 87:3045-3052, 1996

30. Loren AW, Chow E, Jacobsohn DA, et al: Pregnancy after hematopoietic cell transplantation: A report from the late effects working committee of the Center for International Blood and Marrow Transplant Research (CIBMTR). Biol Blood Marrow Transplant 17: 157-166, 2011

31. Sun $C L$, Francisco $L$, Baker $K S$, et al: Adverse psychological outcomes in long-term survivors of hematopoietic cell transplantation: A report from the Bone Marrow Transplant Survivor Study (BMTSS). Blood 118:4723-4731, 2011

32. Syrjala $K L$, Langer $S L$, Abrams JR, et al: Recovery and long-term function after hematopoietic cell transplantation for leukemia or lymphoma. JAMA 291:2335-2343, 2004

33. Sorror ML, Logan BR, Zhu X, et al: Prospective validation of the predictive power of the Hematopoietic Cell Transplantation Comorbidity Index: A Center for International Blood and Marrow Transplant Research Study. Biol Blood Marrow Transplant 21: 1479-1487, 2015

34. Lacasse $\mathrm{Y}$, Montori VM, Lanthier $\mathrm{C}$, et al: The validity of diagnosing chronic obstructive pulmonary disease from a large administrative database. Can Respir J 12:251-256, 2005

35. Lee DS, Donovan L, Austin PC, et al: Comparison of coding of heart failure and comorbidities in administrative and clinical data for use in outcomes research. Med Care 43:182-188, 2005

36. Pine $M$, Jordan $H S$, Elixhauser $A$, et al: Enhancement of claims data to improve risk adjustment of hospital mortality. JAMA 297:71-76, 2007

37. Leal J, Laupland KB: Validity of electronic surveillance systems: A systematic review. J Hosp Infect 69:220-229, 2008

38. Skull SA, Andrews RM, Byrnes GB, et al: ICD10 codes are a valid tool for identification of pneumonia in hospitalized patients aged $>$ or $=65$ years . Epidemiol Infect 136:232-240, 2008 


\section{Affiliations}

Eric J. Chow, Kara L. Cushing-Haugen, Guang-Shing Cheng, Michael Boeckh, Stephanie J. Lee, Wendy M. Leisenring, Paul J. Martin, Beth A. Mueller, Stephen M. Schwartz, and K. Scott Baker, Fred Hutchinson Cancer Research Center; Eric J. Chow, Guang-Shing Cheng, Michael Boeckh, Stephanie J. Lee, Paul J. Martin, Beth A. Mueller, Stephen M. Schwartz, and K. Scott Baker, University of Washington, Seattle, WA; and Nandita Khera, Mayo Clinic, Scottsdale, AZ.

\section{Support}

Funded by grants from the National Institutes of Health (CA151775, CA167451, CA18029). A large portion of the data provided by the Washington State Cancer Registry was collected under contracts N01-CN-67009, N01-PC-35142, and HHSN26120130012I from the National Cancer Institute's Surveillance, Epidemiology, and End Results program.

\section{New Look. Expanded Clinical Content.}

Journal of Oncology Practice (JOP) has a revised design and layout, and expanded clinical content. JOP has changed its design. Check out the new cover, with original illustrations for each issue, and turn the pages to see a brand new layout.
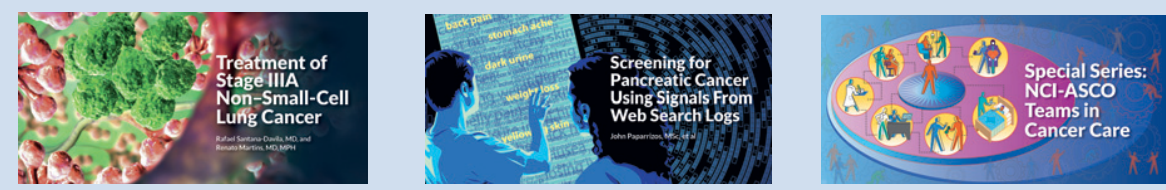

Visit http://jop.ascopubs.org 
AUTHORS' DISCLOSURES OF POTENTIAL CONFLICTS OF INTEREST

Morbidity and Mortality Differences Between Hematopoietic Cell Transplantation Survivors and Other Cancer Survivors

The following represents disclosure information provided by authors of this manuscript. All relationships are considered compensated. Relationships are self-held unless noted. I = Immediate Family Member, Inst = My Institution. Relationships may not relate to the subject matter of this manuscript. For more information about ASCO's conflict of interest policy, please refer to www.asco.org/rwc or ascopubs.org/jco/site/ifc.

Eric J. Chow

No relationship to disclose

Kara L. Cushing-Haugen

No relationship to disclose

\section{Guang-Shing Cheng}

Consulting or Advisory Role: Gilead Sciences

Michael Boeckh

No relationship to disclose

Nandita Khera

Consulting or Advisory Role: Novartis Pharmaceuticals

Stephanie J. Lee

Honoraria: Mallinckrodt

Consulting or Advisory Role: Bristol-Myers Squibb, Kadmon, Amgen

Travel, Accommodations, Expenses: Mallinckrodt

\section{Wendy M. Leisenring \\ Research Funding: Merck}

Paul J. Martin

Consulting or Advisory Role: Pfizer, Incyte, Huron, Enlivex Therapeutics, Janssen

Research Funding: Fresenius Biotech

Beth A. Mueller

Stock or Other Ownership: AstraZeneca (I)

Stephen M. Schwartz

No relationship to disclose

K. Scott Baker

No relationship to disclose 